Boise State University

ScholarWorks

$5-30-2012$

Three-Dimensional Stochastic Estimation of Porosity Distribution:

Benefits of Using Ground-Penetrating Radar Velocity Tomograms in Simulated-Annealing-Based or Bayesian Sequential Simulation Approaches

Baptiste Dafflon

Boise State University

Warren Barrash

Boise State University 


\title{
Three-dimensional stochastic estimation of porosity distribution : Benefits of using ground-penetrating radar velocity tomograms in simulated-annealing-based or Bayesian sequential simulation approaches
}

\author{
B. Dafflon ${ }^{1,2}$ and W. Barrash ${ }^{1}$ \\ Received 13 May 2011; revised 12 March 2012; accepted 17 April 2012; published 30 May 2012.
}

[1] Estimation of the three-dimensional (3-D) distribution of hydrologic properties and related uncertainty is a key for improved predictions of hydrologic processes in the subsurface. However it is difficult to gain high-quality and high-density hydrologic information from the subsurface. In this regard a promising strategy is to use highresolution geophysical data (that are relatively sensitive to variations of a hydrologic parameter of interest) to supplement direct hydrologic information from measurements in wells (e.g., logs, vertical profiles) and then generate stochastic simulations of the distribution of the hydrologic property conditioned on the hydrologic and geophysical data. In this study we develop and apply this strategy for a 3-D field experiment in the heterogeneous aquifer at the Boise Hydrogeophysical Research Site and we evaluate how much benefit the geophysical data provide. We run high-resolution 3-D conditional simulations of porosity with both simulated-annealing-based and Bayesian sequential approaches using information from multiple intersecting crosshole gound-penetrating radar (GPR) velocity tomograms and neutron porosity logs. The benefit of using GPR data is assessed by investigating their ability, when included in conditional simulation, to predict porosity log data withheld from the simulation. Results show that the use of crosshole GPR data can significantly improve the estimation of porosity spatial distribution and reduce associated uncertainty compared to using only well log measurements for the estimation. The amount of benefit depends primarily on the strength of the petrophysical relation between the GPR and porosity data, the variability of this relation throughout the investigated site, and lateral structural continuity at the site.

Citation: Dafflon, B., and W. Barrash (2012), Three-dimensional stochastic estimation of porosity distribution: Benefits of using ground-penetrating radar velocity tomograms in simulated-annealing-based or Bayesian sequential simulation approaches, Water Resour. Res., 48, W05553, doi:10.1029/2011WR010916.

\section{Introduction}

[2] Detailed knowledge of the distribution of parameters controlling flow and transport processes in aquifers is a key prerequisite for realistic simulation of groundwater flow and contaminant transport, and thus for sustainable management and effective remediation of groundwater resources. Estimation of aquifer parameters is difficult, however, given the challenges with direct sampling of the subsurface, and the practical limitations on capturing three-dimensional (3-D) spatial structure. In this regard one promising

\footnotetext{
${ }^{1}$ Center for Geophysical Investigation of the Shallow Subsurface, Department of Geosciences, Boise State University, Boise, Idaho, USA.

${ }^{2}$ Now at Lawrence Berkeley National Laboratory, Berkeley, California, USA.

Corresponding author: B. Dafflon, Lawrence Berkeley National Laboratory, 1 Cyclotron Rd., Berkeley, CA 94720, USA. (baptiste.dafflon@ gmail.com)
}

C2012. American Geophysical Union. All Rights Reserved. 0043-1397/12/2011WR010916 strategy is to use high-resolution geophysical data (that are relatively sensitive to variations of a hydrologic parameter of interest) to supplement direct hydrologic information from measurements in wells (e.g., logs, vertical profiles) and then generate stochastic simulations of the distribution of the hydrologic property conditioned on the hydrologic and geophysical data. Geophysical data can provide information at spatial scales and locations that are unattainable with conventional hydrologic measurement techniques, given a functional or statistical relation between geophysical and hydrologic parameters [e.g., Hubbard and Rubin, 2005]. In this way geophysical data have successfully assisted hydrologic investigations with (1) mapping of hydrogeologically relevant structures [e.g., Beres et al., 1995; Asprion and Aigner, 1999; Barrash and Clemo, 2002], (2) hydrologic parameter estimation [e.g., Hyndman et al., 2000; Chen et al., 2001; Kowalsky et al., 2005; Linde et al., 2006; Paasche et al., 2006; Harp et al., 2008; Dafflon et al., 2010; Straface et al., 2011], and (3) visualization of subsurface processes by monitoring temporal hydrologic changes in the subsurface [e.g., Kemna et al., 2002; 
Day-Lewis et al., 2003; Singha and Gorelick, 2005; Johnson et al., 2007].

[3] Integration of geophysical and hydrologic data with stochastic simulation approaches, such as sequential Gaussian and Bayesian approaches and Monte-Carlo (MC)-type methods [e.g., Deutsch, 2002; Kelkar and Perez, 2002], produce multiple realizations of a target parameter that are consistent with available measurements and spatial structure information. An advantage of such approaches is that the set of simulated realizations represents the joint uncertainty in our knowledge of subsurface properties. For example sequential Gaussian simulation [e.g., Deutsch and Journel, 1998] has been used successfully to integrate crosshole seismic and geostatistical information by Hyndman et al. [2000], and also by McKenna and Poeter [1995] for generating indicator simulations based on crosshole seismic tomography and borehole logging information. Bayesian approaches [e.g., Gelman et al., 2003] have been applied to integrate diverse geophysical and hydrologic data [e.g., Ezzedine et al., 1999; Chen et al., 2001], and can be included within a sequential simulation approach [e.g., Scheibe and Chien, 2003]. Recently Dubreuil-Boisclair et al. [2011] successfully used Bayesian sequential simulation to integrate crosshole GPR velocity and amplitude tomograms with well measurements to image the hydraulic conductivity distribution. Also, another simulation strategy that is particularly flexible for integrating various data sets, constraints, and petrophysical relations is the optimization of a parameter realization through a Monte-Carlo (MC) perturbation process for a given objective function [e.g., Deutsch and Wen, 2000; Kelkar and Perez, 2002; Tronicke and Holliger, 2005; Dafflon et al., 2009a]. Among the MC methods, a simulated-annealing (SA)-based approach developed by Dafflon et al. [2009a] generates realistic spatial distributions of porosity using crosshole GPR tomograms and porosity log data.

[4] The objective of this study is to develop improved stochastic integration techniques for 3-D estimation of the porosity distribution, and to demonstrate the approaches with data from the Boise Hydrogeophysical Research Site (BHRS). We evaluate added benefit from using multidirectional crosshole GPR velocity tomograms [Dafflon et al., 2011a] with porosity log data [Barrash and Clemo, 2002] for the estimation of the 3-D distribution of porosity and associated uncertainty. The basis to relate GPR velocity to porosity is the strong sensitivity of GPR velocity to soil water content, which is equivalent to porosity in the saturated zone [e.g., Schön, 2004]. High-resolution conditional simulations of the porosity spatial distribution using these data are generated using both SA-based [e.g., Dafflon et al., 2009a] and Bayesian simulation approaches [e.g., DubreuilBoisclair et al., 2011]. We selected these methods because of their demonstrated success compared to other approaches in 2-D conditional simulations of hydrologic parameter distributions using data similar to those used in this study [Dafflon et al., 2009b; Dubreuil-Boisclair et al., 2011]. Here these approaches are adapted to 3-D and to the general case of variably spaced data (as are available at the BHRS and many sites). The benefit added by including crosshole GPR tomograms in the stochastic estimation of the porosity spatial distribution is assessed by evaluating how well porosity values are predicted at locations where porosity log data are withheld. This is done for two cases where the withheld porosity log is located: (1) at a crosshole GPR profile, so the full available data set, with the exception only of the withheld porosity $\log$, is used to condition the 3-D simulation; and (2) where no data are available so all the existing crosshole profiles intersecting the withheld porosity $\log$ are also withheld for this estimation process. For both cases we do this for two wells having locally variable stratigraphy.

[5] Developing an effective integration approach to estimate the porosity spatial distribution in unconsolidated aquifers is important because porosity heterogeneity can play a significant role in transport behavior [Hassan, 2001; Hu et al., 2009; Dafflon et al., 2011b; Li et al., 2012]. Also, although the spatial distribution of hydraulic conductivity generally is much more difficult to estimate than porosity, these parameters commonly exhibit some degree of similarity with regard to their spatial variability and/or spatial correlation [e.g., Hubbard et al., 2001; Scheibe and Chien, 2003].

[6] In this paper we first describe the simulation approaches used to perform 3-D conditional simulations. Next, we briefly describe the porosity log data and GPR velocity tomograms from the BHRS, and the conditional petrophysical relations and geostatistical models used in the simulation process. Then we evaluate the benefit gained from including crosshole GPR data in the simulation process by predicting porosity with several approaches. Finally, we discuss improvements achieved in imaging hydrostratigraphic units at the BHRS.

\section{Stochastic Approaches}

\subsection{SA-based Optimization Approach}

[7] SA is a directional MC-type optimization procedure [Deutsch and Wen, 1998; Day-Lewis et al., 2000; Kelkar and Perez, 2002; Tronicke and Holliger, 2005; Dafflon et al., 2009a] that involves the repeated perturbation of a random field in order to satisfy a multicomponent global objective function. In this study we use the SA-based conditional simulation approach of Dafflon et al. [2009a] which was developed to simulate hydrologic parameter fields by assimilating both larger-scale subsurface structures (provided by geophysical data) and smaller-scale fluctuations (provided by well log data and spatial correlation functions). This approach consists of: (1) generation of an initial realization of the target parameter (e.g., porosity), (2) random selection and perturbation of a cell in the model by drawing from a probability distribution of the target parameter given the available data at that location (e.g., using conditional petrophysical distributions), (3) updating the objective function (e.g., spatial correlation functions), (4) accepting the perturbation if the objective function is lowered according to a Boltzmann-type exponential probability function, (5) repetition of steps (2) to (4) until the objective function is satisfied. The initial realization is generated by performing the second step for each cell in the model to ensure that each initial realization is different.

[8] Here we adapted the approach of Dafflon et al. [2009a] to 3-D and to variably spaced data. To this end, the generation of the initial realization (1) and each perturbation (2) depends on the available data at the considered cell. That is, if crosshole GPR data are available at a given cell, the new value is drawn randomly from a probability distribution of porosity given the available GPR data at the 
cell location using the conditional petrophysical distribution developed from collocated GPR and porosity data at the wells (also see discussion in section 4.1). Next, at and near the boreholes the probability distribution of the porosity is more tightly constrained with a conditional expectation equal to the porosity log data and a relatively small standard deviation defining its uncertainty. Finally, at cells where no data are available the conditional expectation is obtained through kriging the log data and the conditional expected porosity values inferred from the crosshole GPR data through the conditional petrophysical distribution. The variance of the porosity probability distribution at such cells is set equal to the variance of all the log data present in a depth interval centered on the simulated location and equal to the range of the vertical spatial correlation function of the porosity. We do this instead of using the kriging variance because it is difficult to assess the true variance at these locations, and so we prefer to allow relatively large variability where no data are available (i.e., to overestimate versus underestimate the true uncertainty).

[9] The objective function (3) is defined by matching a parametric spatial correlation function in $\mathrm{x}, \mathrm{y}$ and $\mathrm{z}$ directions. Dafflon et al. [2009a] have shown that these correlation models need to be considered only at "relatively small" lags where the GPR data fail to capture the heterogeneity and where variability can be estimated relatively well from available porosity log data, while at large scales the variability is constrained only by GPR data. This is similar to assigning a very high uncertainty to the porosity spatial correlation function at large lags. Finally, a last parameter that can be important in the optimization process is the "temperature" parameter in the Boltzmann-type exponential probability function [e.g., Dafflon et al., 2009a], which controls the extent to which perturbations that do not lower the objective function are accepted (4). In this study, due to the relative simplicity of the objective function and based on some preliminary tests, the temperature parameter does not significantly influence the process and therefore has been set relatively low.

\subsection{Bayesian Sequential Simulation}

[10] The Bayesian sequential simulation approach is mainly based on traditional sequential Gaussian simulation [e.g., Deutsch and Journel, 1998] with the added use of Bayesian formula [e.g., Chen et al., 2001; Gelman et al., 2003]. The Bayesian methodology has been used widely with success for updating prior knowledge with additional conditional sources of information [e.g., Ezzedine et al., 1999; Chen et al., 2001; Linde et al., 2007], and especially in the context of Bayesian sequential simulations [Gastaldi et al., 1998; Scheibe and Chien, 2003; Dubreuil-Boisclair et al., 2011]. In this approach each new simulated value is drawn from a probability distribution obtained by updating a prior distribution of a target parameter into a posterior distribution given additional data at a given location and using estimates of the conditional petrophysical relation between collocated geophysical and hydrologic data. In a recent study by Dubreuil-Boisclair et al. [2011] the 2-D hydraulic conductivity distribution between two boreholes was estimated with Bayesian sequential simulation using collocated results from a GPR velocity and attenuation tomogram and hydraulic conductivity measurements at the wells to estimate multivariable relations between hydraulic conductivity, electrical conductivity, and permittivity with a kernel probability function [e.g., Wand and Jones, 1995]. Their Bayesian simulation approach showed clear improvement compared to other approaches such as a sequential co-simulation approach.

[11] The 3-D Bayesian sequential simulation approach used here consists of two stages. The first stage is performed only at cells located along crosshole GPR profiles. The process consists of (1) selecting a cell that has not been visited yet, (2) applying simple kriging under a Gaussian assumption using all the available values of porosity to estimate the expected mean and variance that define the prior distribution at the visited cell, (3) defining the likelihood distribution obtained from both the available crosshole GPR data at the selected cell and the conditional petrophysical distribution inferred from the collocated crosshole GPR and porosity log data at the wells, (4) inferring the posterior probability distribution by applying Bayes' rule to update the prior distribution with the likelihood distribution (by calculating their product), (5) drawing a porosity value from the posterior probability distribution for the selected cell and adding it to the available porosity data before visiting the next cell, and (6) repeating the above steps until all the selected cells have been visited. It is worth noting that the conditional petrophysical distribution used to estimate the likelihood distribution (3) is identical to the one used in the SA approach (see also section 4.1).

[12] The second step of our approach is to perform standard sequential Gaussian simulation in all the remaining cells that do not contain any data. In fact this stage is identical to the previous one except that no likelihood function is available and thus the prior distribution alone defines the posterior probability distribution from which a value of porosity is drawn. Finally we note that, although we use simple kriging in this paper, we have conducted additional investigations that show the use of ordinary kriging does not change the results of this study significantly.

\section{Hydrogeologic Field Site}

\subsection{Well Measurements}

[13] The BHRS is a hydrologic and geophysical field research site located near Boise, Idaho, USA. The shallow unconfined aquifer at the site resides within an approximately 20-m-thick layer of sediments consisting of coarse, unconsolidated, fluvial deposits [Barrash and Clemo, 2002; Barrash and Reboulet, 2004] with minimal fractions of silt and clay, and is underlain by a layer of red clay. At this site 18 wells have been emplaced in a configuration to facilitate a wide-variety of hydrologic and geophysical testing [Barrash et al., 1999]. All of the wells were cased with 4-in PVC well screen and were carefully completed to minimize the disturbance of the surrounding formation. The well field consists of 13 wells in the central area $(\sim 20 \mathrm{~m}$ in diameter) and five boundary wells about 10 to $35 \mathrm{~m}$ from the central area (Figure 1). The general design of the 13 inner wells is a central well (A1) surrounded by two concentric rings of six wells each (B1-B6 and C1-C6, respectively). The distances between these wells vary between 2.6 and $8.6 \mathrm{~m}$.

[14] Porosity values in each borehole were estimated at $0.06 \mathrm{~m}$ intervals from the measured neutron-log count rate 


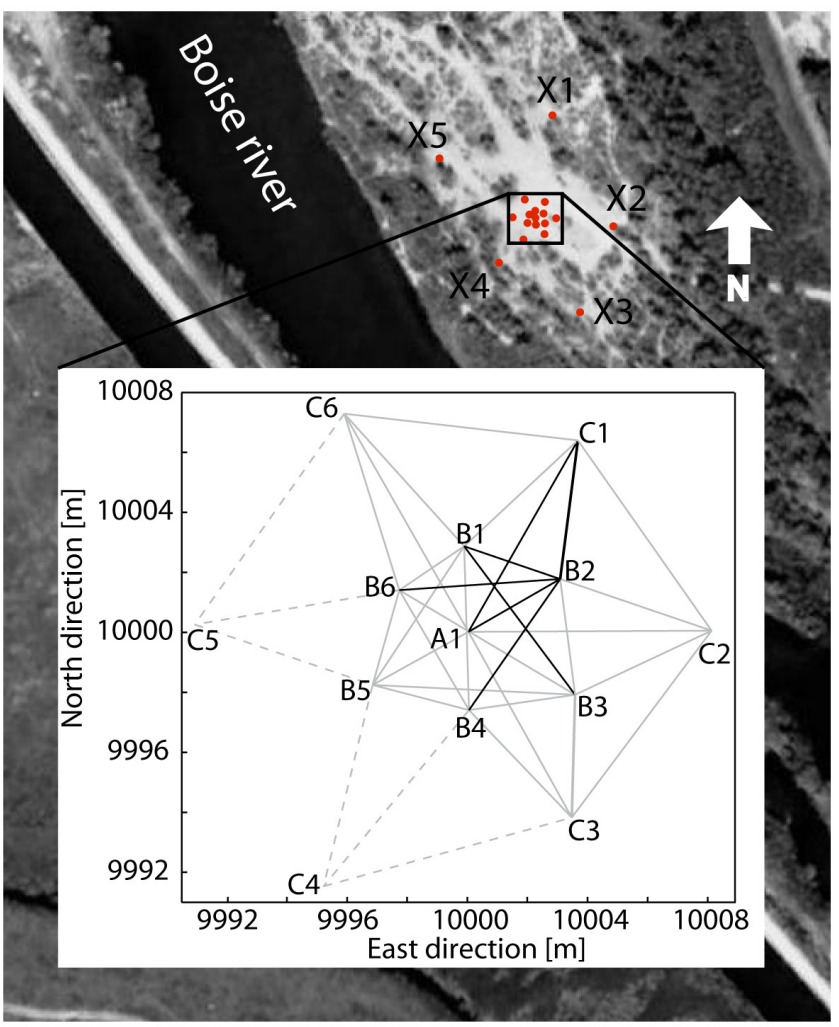

Figure 1. Aerial view of the BHRS and detailed map of the central area wells with lines locating crosshole GPR tomograms. Joint inversion of multiple GPR profiles to reconstruct a multidirectional model of GPR velocity was performed for both 31 (continuous gray and black lines) and seven data sets together (black lines).

[Barrash and Clemo, 2002] through a petrophysical transform [Hearst and Nelson, 1985] calibrated with measurements of porosity in similar environments [Barrash and Clemo, 2002]. Based on these porosity logs Barrash and Clemo [2002] identified five units with distinct spatial distribution, mean, and variance. The fitted Gaussian functions to the porosity distributions of Units 1 to 5 have means equal to $0.18,0.24,0.172,0.224$ and 0.425 , respectively, and standard deviations equal to $0.022,0.038,0.024,0.05$ and 0.055 , respectively. Unit 5 is a high porosity sand channel that thickens toward the Boise River and pinches out in the center of the well field. Units 1-4 are a sequence of conglomerates with gravel and cobble framework and sand to pebble matrix in the interstices of the framework. More recently capacitive electrical conductivity measurements have identified a subunit (Unit 2b) that is present in all the wells shown in the inset of Figure 1 except wells B1, B3, $\mathrm{C} 1$ and C2 [Mwenifumbo et al., 2009]. Four of the five main units (i.e., Units 1-4) occur in the depth interval discussed in this paper (Figure 2).

\subsection{Crosshole GPR Tomograms}

[15] A total of 38 crosshole GPR data sets were acquired from 1998 to 2000 between the 13 wells in the central area of the BHRS (Figure 1) using a Mala Ramac GPR system with antennas having a nominal center frequency in air of $250 \mathrm{MHz}$. For this study we used 31 crosshole data sets

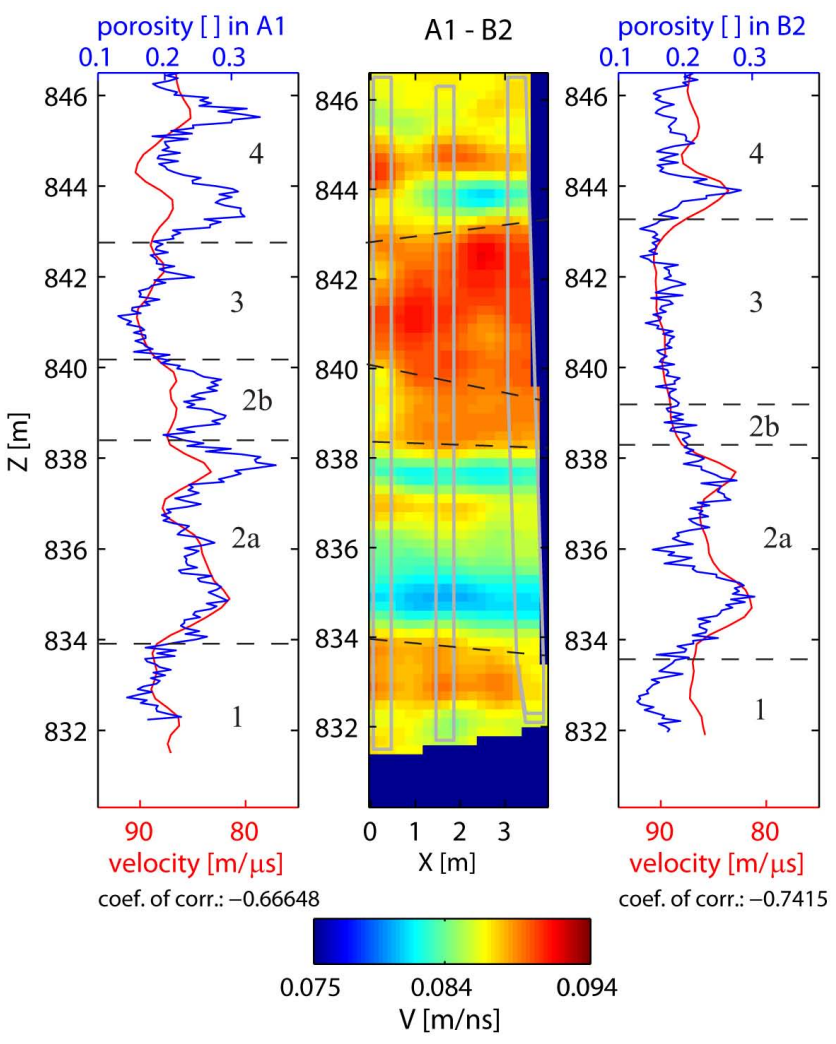

Figure 2. Velocity tomogram for crosshole profile between A1-B2 from inversion of seven crosshole GPR data sets together (modified from Dafflon et al. [2011a]). Intersections of other profiles through the A1-B2 profile are delineated (gray). The correlation between velocity and porosity-log data at the borehole location is given at the bottom of each log comparison panel. For comparison with stratigraphy at the BHRS, contacts between Units 1 to 4 are identified in the porosity logs [Barrash and Clemo, 2002; Mwenifumbo et al., 2009].

between 11 wells. All the data have a common depthsampling interval of approximately $0.2 \mathrm{~m}$ in the transmitter and receiver boreholes and cover the saturated zone between 846.5 and $832 \mathrm{~m}$ elevation. Antenna positions were corrected to account for borehole deviations using measurements from a magnetic deviation logging survey in 2010. Travel times of the direct transmitted wavefield for each crosshole GPR data set were determined using a semiautomated picking procedure.

[16] GPR velocity tomograms used in this study were obtained by jointly inverting intersecting crosshole GPR travel time data sets [Dafflon et al., 2011a]. In this method, first all transmitter and receiver coordinates, and coordinates of the related profile intersections, are projected in 2-D. Then the tomographic kernel matrix, regularization matrix, and model vector are constructed such that (1) common cells are considered where profiles intersect and (2) all profiles are considered together in a single expanded tomographic system. The velocity is inverted for the set of all 2-D cells through ray-based inversion using a conjugategradient-based least-squares approach [e.g., Scales, 1987] and accounting for possible errors in the crosshole GPR 
survey geometry, and for antenna transmission behavior and locations [Irving et al., 2007; Dafflon et al., 2011a].

[17] The above inversion strategy has been shown to be a robust procedure for two different cases at the BHRS: (1) 31 profiles, or all the available data with the exception of the profiles obtained using wells C4 and C5, and (2) a subset of seven profiles of higher-than-average quality in a correspondingly limited area of the whole data set (Figure 1). The GPR velocity tomograms inverting seven profiles together (from Dafflon et al. [2011a]) and shown here for A1-B2 (Figure 2): (1) successfully image the major stratigraphic units and their contacts at the BHRS, and (2) confirm the variability differences within and between units evident in porosity data [Barrash and Clemo, 2002], including recognition of smaller-scale subfacies structures. The correlation coefficients between the collocated GPR velocities and porosity data at the 11 wells used in this study (Figure 1) range between -0.32 and -0.79 with a mean of -0.57 . We note that there is no link between the values of the correlation coefficient and the number of GPR crosshole profiles intersecting at each well location [Dafflon et al., 2011a]. With few exceptions, these correlations can be considered as significant given that GPR data have a more limited spatial resolution than, and are not able to define small-scale heterogeneity near boreholes as well as, the porosity measurements (Figure 2). Also these correlation coefficients are known to be relatively decreased by the presence of subunit $2 \mathrm{~b}$ which is less well imaged by the GPR data due to anomalous conductivity/dielectric petrophysical behavior not dominated by water-saturated porosity [Mwenifumbo et al., 2009; Dafflon et al., 2011a].

\section{Conditional Petrophysical Distribution and Spatial Correlation Function}

\subsection{Conditional Petrophysical Distribution From the Collocated Data}

[18] Both SA-based and Bayesian sequential simulation approaches require that GPR velocity be linked to porosity through a conditional petrophysical distribution. Although a simple approach for hydrogeophysical applications is to relate parameters using laboratory-derived petrophysical relationships, such relationships may only be valid at the small scale and can encounter problems when they are used to "convert" geophysical images to subsurface hydrologic properties. Here we prefer to build a site-specific conditional petrophysical distribution of porosity given geophysical data using collocated data sets at boreholes. Clearly, in this case, the quality of the estimated conditional petrophysical distribution is dependent upon the number and quality of collocated data [e.g., Ezzedine et al., 1999; Chen et al., 2001; Bachrach, 2006; Paasche et al., 2006]. Although this approach does not address the fact that the velocity-porosity relation may in reality vary in space due to differences in tomographic resolution throughout the image plane [e.g., Day-Lewis and Lane, 2004; Moysey et al., 2005], it avoids significant complications and ambiguities associated with accurately estimating the spatially variable relationship, and has been shown to be a reasonable approach to predicting porosity in saturated alluvial aquifers [Dafflon et al., 2009a].

[19] In this study, we estimate the site specific conditional petrophysical distribution between collocated GPR velocity and porosity data with a parametric approach [e.g., Corbeanu et al., 2002; Dafflon et al., 2009a]. This choice is supported by the relatively strong negative correlation between velocity and porosity, the somewhat limited size of the data set for nonparametric approaches, and the existing homoscedasticity in the conditional petrophysical distribution (Figure 3 ). Based on preliminary tests, we assume a linear relationship with constant variance between GPR velocity and the natural logarithm of porosity; that is we assume that the conditional expectation of the natural logarithm of porosity $E(\ln (\phi) \mid \nu)$ given the velocity $v$ is obtained through linear regression by minimizing the mean square error of the prediction. The variance $\sigma^{2}(\ln (\phi) \mid \nu)$ is then obtained from the distribution of the residuals between predicted and measured natural logarithms of porosity. Based on Figure 3, we assume that the conditional petrophysical distribution is approximately Gaussian, and therefore we only need to know the mean and variance to draw values from the distribution. In this regard the natural logarithm of porosity is considered to have a more-Gaussian distribution of residuals, and also has the advantage of avoiding negative porosity values in the simulation process. Furthermore, although uncertainty in the GPR velocity tomogram is not directly included in this approach, its effect is considered indirectly by the use of a field-based (i.e., in situ) conditional petrophysical distribution-which implies that the full range of porosity values related to each smoothed velocity value is influencing the petrophysical relationship and distribution (Figure 3).

\subsection{Spatial Correlation Function}

[20] The SA- and Bayesian sequential simulation processes both require a geostatistical model in the $\mathrm{x}-, \mathrm{y}-$ and

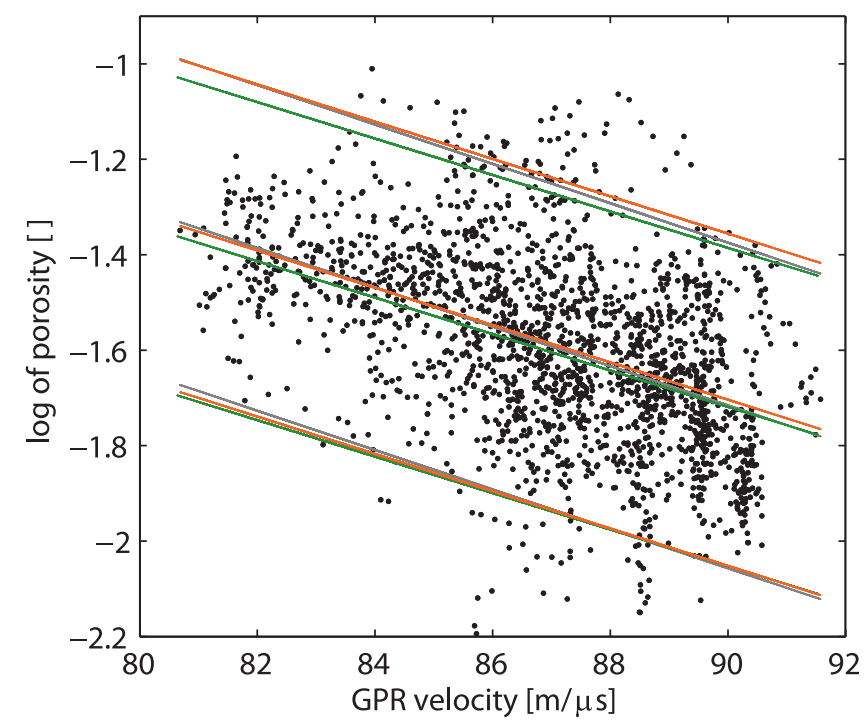

Figure 3. Scatterplot of GPR velocity versus the natural logarithm of porosity at wells A1, B1, B2, B3, B4, B6, and $\mathrm{C} 1$. The central lines denote the conditional expectation $\mathrm{E}(\ln (\phi) \mid v)$ assuming a linear relationship and minimizing the squared error when using: all the wells (black); all wells except B2 (red); and all wells except A1 (green). The peripheral lines on the sides indicate two times the conditional standard deviation $\sigma(\ln (\phi) \mid v)$. 
z-directions to enforce structural variability and continuity throughout the realization. In this study we estimated the spatial correlation function used for the geostatistical model by fitting an exponential model [e.g., Goovaerts, 1997; Deutsch and Journel, 1998] to the experimental spatial correlation function calculated from the porosity log data. The geostatistical model is characterized by a vertical range of $4 \mathrm{~m}$ based on porosity log data from the full aquifer thickness; this value is similar to the vertical range estimated for the full aquifer thickness at the BHRS previously by another approach [Barrash and Clemo, 2002] and does not produce significant variations in the simulations when changed within a realistic range of uncertainty.

[21] The horizontal spatial correlation function was inferred from the porosity logs assuming an isotropic model because there is little evidence for horizontal anisotropy. The uncertainty is clearly higher in the horizontal than vertical direction because of more limited data in the horizontal direction. From the porosity logs we estimated a realistic interval for the range of the lateral spatial correlation function to be between 12 and $24 \mathrm{~m}$, which is consistent with findings of other studies for lateral geostatistics of site-wide hydrologic properties at the BHRS [Barrash and Clemo, 2002; Cardiff et al., 2011]. Although the uncertainty of the horizontal range could be included directly in the simulation process, here we only present results with a range of $24 \mathrm{~m}$ because the findings of this study were not changed much when the range was varied between 12 and $24 \mathrm{~m}$.

\section{Results}

\subsection{Impact of Including GPR Data in Porosity Simulations}

\subsubsection{Predicting Porosity Along GPR Profiles}

[22] To assess the benefit of using crosshole GPR data to supplement well log data to predict porosity at locations where only crosshole GPR data are available, a porosity $\log$ is intentionally withheld from the simulation process and then later compared with the prediction at the withheld location. This is done for two cases: withholding well B2 and then A1. The GPR data consist of seven profiles inverted together (Figure 1), among which some include well A1 or B2. For each case, collocated data for GPR velocity and the natural logarithm of porosity are used to infer the conditional petrophysical distribution, which does not change much when one porosity log (either well B2 or A1) is withheld from the process (Figure 3 ). Then 100 realizations of porosity are generated for each case (1) using supporting GPR data with SA-based (Figures 4a and 4d) and Bayesian sequential simulation (Figures $4 \mathrm{~b}$ and $4 \mathrm{e}$ ) approaches, and (2) using porosity log data only with a sequential simulation approach (Figures $4 \mathrm{c}$ and $4 \mathrm{f}$ ). For each case we also show the absolute residual and the distribution of the correlation coefficient and of the mean absolute residual between each simulated and measured "true" porosity $\log$.

[23] Use of GPR data with either the SA-based (Figure $4 a)$ or the Bayesian sequential (Figure 4b) approach significantly improves the prediction of porosity at well B2 compared to using only the porosity $\log$ data (Figure $4 \mathrm{c}$ ) as seen by (1) lower residual and relative errors between simulated and true porosity, (2) lower uncertainty in the prediction, and (3) higher correlation coefficient between simulated and "true" porosity. In particular, by including GPR data, the mean correlation coefficient between predicted and "true" porosity logs increased from 0.285 to 0.710 with SA-based simulation and from 0.285 to 0.515 with Bayesian sequential simulation. The only places where GPR data did not clearly improve predictions of porosity are at the tops and bottoms of the tomograms, which are well known to have the largest uncertainty and, commonly, presence of artifacts due to more limited ray coverage.

[24] Prediction of the withheld porosity $\log$ at A1 (Figures $4 \mathrm{~d}-4 \mathrm{f}$ ) also is significantly improved when GPR data are included in the simulation, although less than at B2 in terms of the residuals between simulated and measured data. This is particularly so for depth intervals (1) between $838 \mathrm{~m}$ and $840.2 \mathrm{~m}$ where Unit $2 \mathrm{~b}$ is known to have anomalous petrophysical behavior [Mwenifumbo et al., 2009], and (2) between $843 \mathrm{~m}$ and $844.5 \mathrm{~m}$ where relatively high-porosity lenses occur in Unit 4. However, even though GPR data do not accurately image all variations at A1 compared to B2 (Figure 2), still inclusion of crosshole GPR data in the simulations clearly improves the prediction of porosity at A1 as demonstrated by nearly doubling the correlation coefficients between simulated and true porosity (Figures $4 \mathrm{~d}-4 \mathrm{f}$ ).

\subsubsection{Predicting Porosity Outside Crosshole GPR Profiles}

[25] Here we evaluate the benefit of including GPR data for simulation of the 3-D distribution of porosity at locations where no data (of any kind) are available. Again a porosity $\log$ is withheld from the simulation and then later compared to the predicted porosity at the log's location, but also the GPR profiles through this location are not included in the simulation. Thus, instead of using seven GPR profiles as in section 5.1.1., only two GPR profiles are included (B1-B3 and A1-C1) when B2 is withheld, and five are included when A1 is withheld (B1-B2, C1-B2, B2-B4, B1-B3 and B6-B2). Figure 5 shows the 100 simulated porosity logs and the measured "true" logs at wells B2 (Figures 5a-5c) and A1 (Figures 5d-5f) obtained by integrating GPR data with SA-based (Figures 5a and 5d) and Bayesian sequential (Figures $5 \mathrm{~b}$ and $5 \mathrm{e}$ ) simulations, and by using only the porosity $\log$ with sequential simulation (Figures $5 \mathrm{c}$ and $5 \mathrm{f}$ ).

[26] The use of GPR data in either SA-based (Figures 5a and $5 \mathrm{~d}$ ) or Bayesian sequential (Figures $5 \mathrm{~b}$ and $5 \mathrm{e}$ ) simulation still significantly improves the prediction of porosity at wells $\mathrm{B} 2$ and A1 compared to using only the porosity logs (Figures $5 \mathrm{c}$ and $5 \mathrm{f}$ ), even if no GPR profile intersects the locations of the porosity logs. The mean correlation coefficient between predicted and "true" porosity logs at B2 increased significantly from 0.285 to 0.616 , and from 0.285 to 0.439 by including GPR data in SA-based and Bayesian sequential simulations, respectively. This again indicates the strong contribution of GPR data in constraining the porosity spatial distribution, even at locations where no data are present.

\subsection{Differences Between SA-based and Bayesian Sequential Simulation Approaches}

[27] In this study, the SA-based approach results in better predictions of porosity than the Bayesian sequential simulation approach (Figures 4 and 5). However universal 

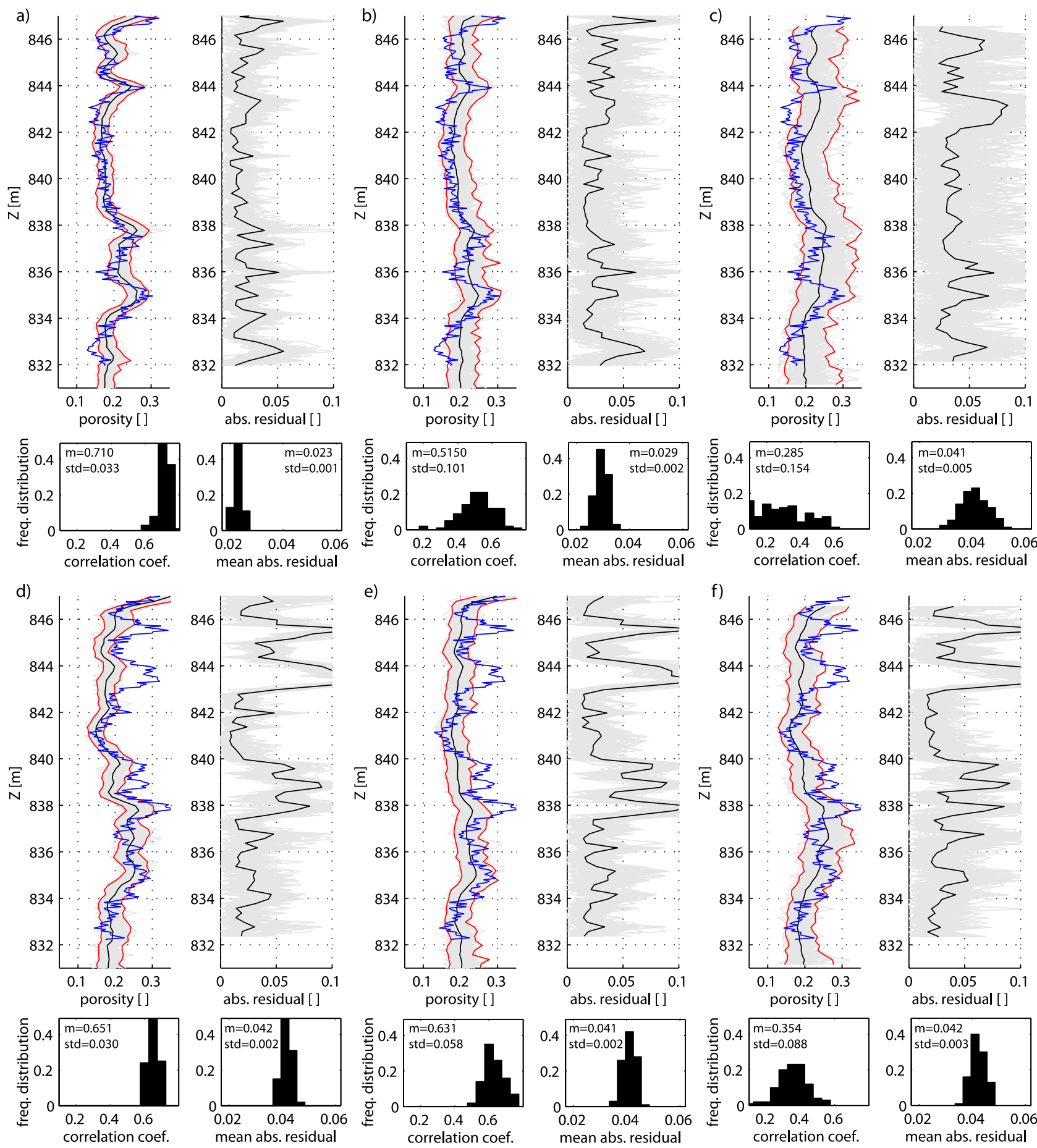

Figure 4. Evaluating predictions of measured porosity values (blue lines) that are withheld from the estimation process and located along crosshole GPR profiles: $(\mathrm{a}-\mathrm{c})$ at well B2 and (d-f) at well A1. This is done by generating 100 3-D realizations (gray lines) of porosity using crosshole GPR and porosity log data in ( $a$ and d) SA-based and (b and e) Bayesian sequential simulation approaches, and (c and f) using only the porosity log data in a sequential simulation approach. The black lines show the means of all the simulations and the red lines show the $95 \%$ intervals of occurrence. Frequency distributions of the correlation coefficients and of the mean absolute residuals between each simulation and the measured data are also shown.

generalizations should not be drawn because relative performance will depend on details of a given system including data sets and relations. The main difference between the approaches is that in the SA-based approach the simulated porosity values at the GPR profiles are only indirectly influenced by porosity logs through the spatial correlation function used in the objective function. With the Bayesian sequential approach however, simulated 

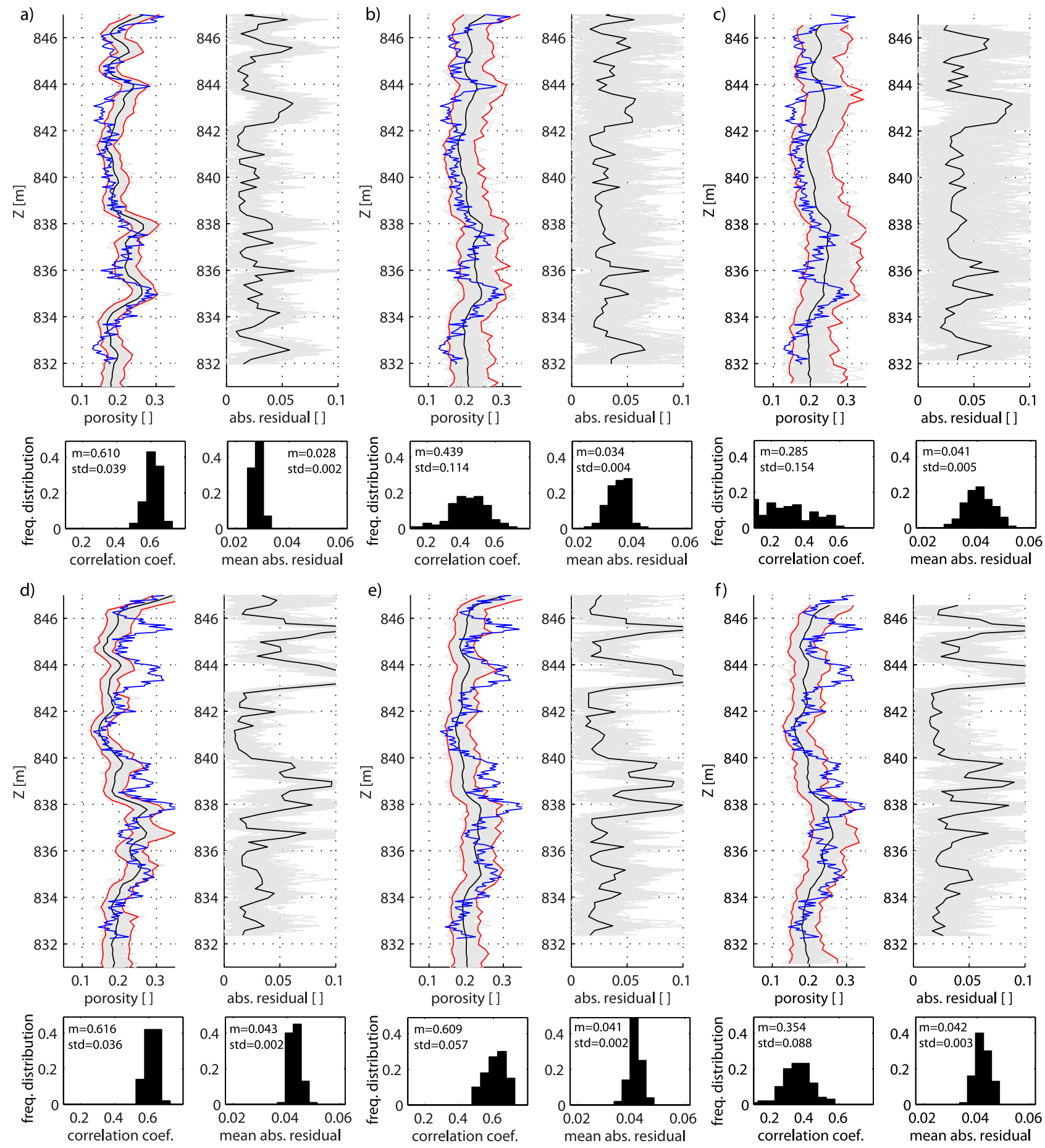

Figure 5. Evaluating predictions of measured porosity values (blue lines) that are withheld from the estimation process and not located along crosshole GPR profiles (produced by removing profiles intersecting at withheld locations): (a-c) at well B2 and (d-f) at well A1. This is done by generating 100 3-D realizations (gray lines) of porosity using crosshole GPR and porosity log data in (a and d) SA-based and ( $b$ and e) Bayesian sequential simulation approaches, and (c and f) using only the porosity log data in a sequential simulation approach. The black lines show the means of all the simulations and the red lines show the $95 \%$ intervals of occurrence. Frequency distributions of the correlation coefficients and of the mean absolute residuals between each simulation and the measured data are also shown. 
porosity values are directly influenced by the porosity logs used to generate the prior distribution through kriging. Doing this tends to create some averaging between the GPR and well log information, and thus the Bayesian simulated porosity field (e.g., Figures $4 \mathrm{~b}$ and $4 \mathrm{e}$ ) can be considered as an intermediate state between the SA-based (e.g., Figures $4 \mathrm{a}$ and $4 \mathrm{~d}$ ) and the sequential (e.g., Figures $4 \mathrm{c}$ and 4f) simulation results. This means the Bayesian sequential simulation approach may give better results where the "kriged" prior distribution from porosity logs (and alreadysimulated data) is reliable and/or where GPR information is less informative (for whatever reasons).

[28] A second difference between the approaches is that the spatial correlation function is applied indirectly in the SA-based approach through a global objective function, but more directly influences each new simulated porosity value in the Bayesian approach. However we did not observe significant structural differences in the results due to this difference in how the spatial correlation function is applied. Indeed, we also note that the experimental spatial correlation functions all show good agreement with the target spatial correlation function, with (Figures $4 \mathrm{a}$ and $5 \mathrm{a}$ ) some fluctuation around it, and (Figures $4 \mathrm{~b}$ and $5 \mathrm{~b}$ ) slightly smaller mean of the spatial correlation ranges.

\subsection{Accounting For Variable Spatial Statistics and/or Conditional Petrophysical Distributions}

[29] One issue about including GPR data in the simulation of the porosity spatial distribution is that the range of the simulated porosity values does not always contain the true porosity $\log$, as for example at A1 (Figures $4 \mathrm{~d}-4 \mathrm{f}$ and Figures $5 \mathrm{~d}-5 \mathrm{f})$. In this regard and in recognition of differences associated with Unit $2 \mathrm{~b}$, which occurs in most wells within about 838 and $840.2 \mathrm{~m}$, we first simulated the porosity spatial distribution independently for three layers, with boundaries at these depths The resulting simulations show that the porosity predictions were slightly improved in this depth interval at well A1 but were decreased in the same depth interval at well B2. In fact some lateral thickness and porosity variations occur between Units $2 \mathrm{~b}$ and 3 [Mwenifumbo et al., 2009] which, in places, are not consistent with thickness of the middle model layer or are not consistently imaged by the GPR tomograms (Figure 2).

[30] A follow-up reconnaissance effort was made to increase the variability between simulations by considering the uncertainty related to wells used to infer the conditional petrophysical distribution. This effort consists of (1) calculating, independently at each well, the likelihood distribution for a range of parameter values describing a linear relationship between collocated GPR velocity and natural logarithm of porosity by grid search [e.g., Dafflon et al., 2010], (2) summing the volume-normalized likelihood distributions obtained at the different wells, and (3) drawing a linear relationship (from the resulting likelihood distribution) to define the conditional petrophysical distribution prior to each new 3-D simulation of the porosity field. For this analysis, we assumed (1) the uncertainty in the measured data to have a Gaussian distribution with a standard deviation of about 0.02 and (2) the constant variance of the conditional distribution to be equal to the maximum variance of the residual observed throughout all the best fitting linear relationships.

[31] Figures $6 \mathrm{a}$ and $6 \mathrm{~b}$ show the likelihood functions obtained for a large range of evaluated slopes and means of natural logarithm of porosity that define linear relationships, while withholding porosity data at well B2 and then A1, respectively. Note that evaluating the mean of natural logarithm of porosity or the intercept is equivalent for a constant mean value of GPR velocity (here equal to $87.07 \mathrm{~m} / \mu \mathrm{s}$ ). From Figure 6 it is clear that the "best" fitting relation
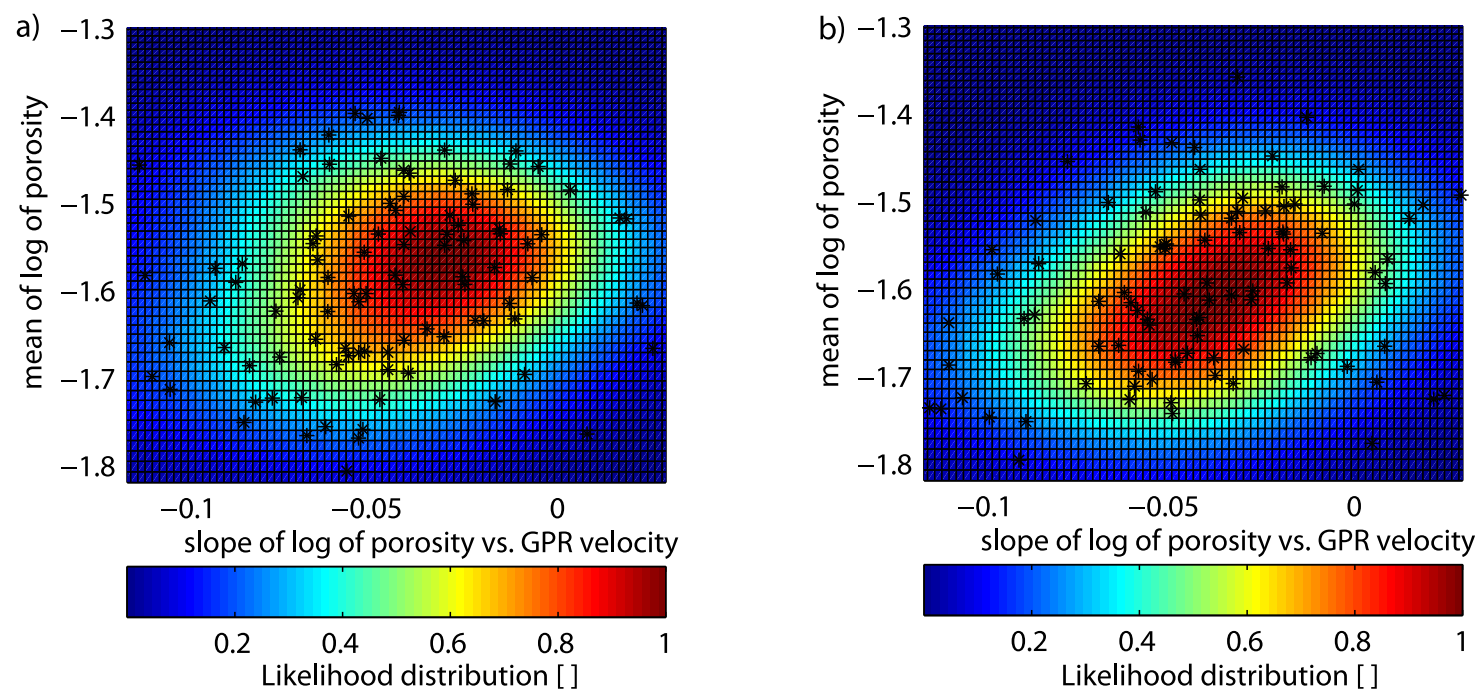

Figure 6. Final likelihood distributions of the slope and mean of the natural logarithm of porosity defining the linear relationship between GPR velocity and the natural logarithm of porosity, and obtained from independent grid searches through the mean square error for each of the wells B1, B3, B4, B6 and C1, and including (a) A1 and (b) B2. Black stars indicate randomly selected values in the distribution that allow definition of 100 conditional petrophysical relationships between GPR velocity and porosity when withholding (Figure 6a) B2 and (Figure 6b) A1, and that were used to perform the simulations shown in Figure 7. 
between GPR velocity and porosity data changes from well to well (sometimes significantly) and thus the shape of the final likelihood function is related to the particular wells used in the analysis. Figure 7 shows porosity at B2 and then A1 from SA-based simulation using GPR data, and
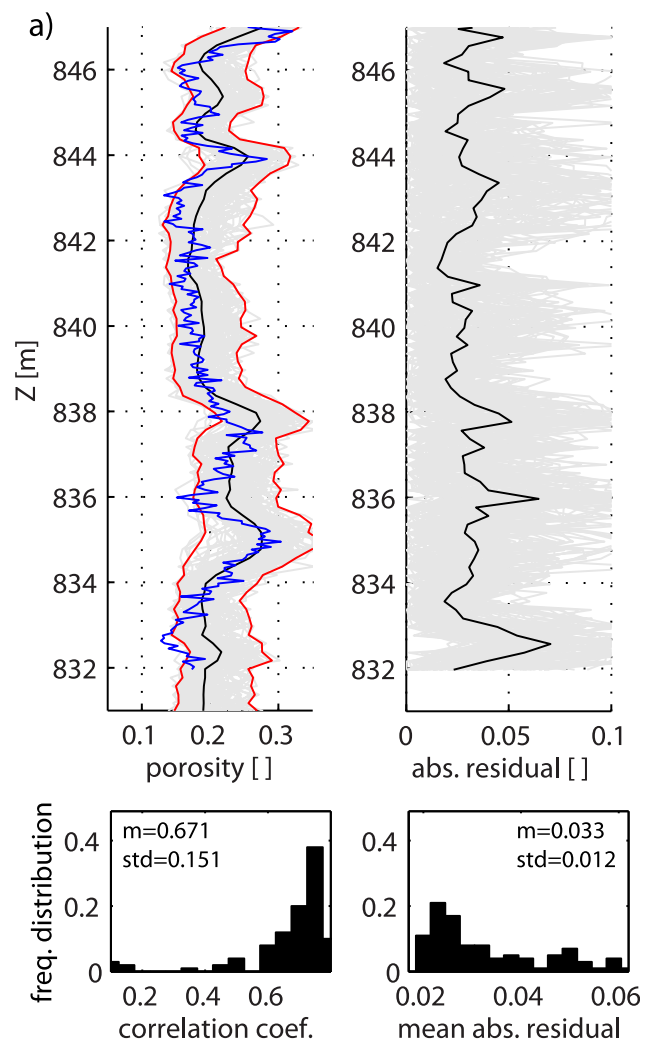

b)
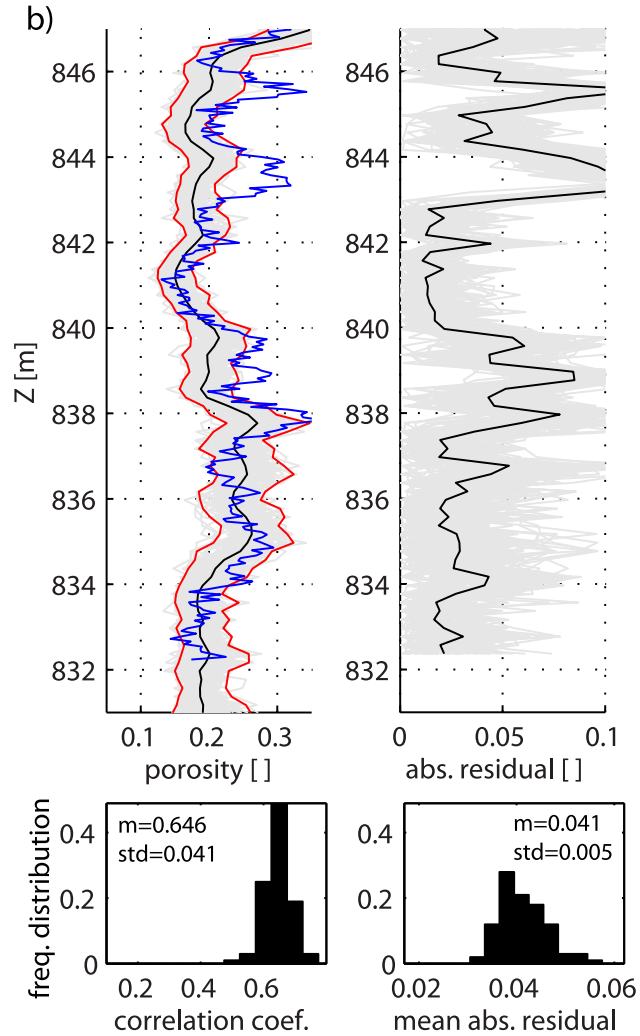

now including the random selection of a conditional petrophysical distribution from the likelihood function shown in Figure 6. Compared to the previous approach (Figures 4a and $4 \mathrm{~d}$ ), doing this allows us to increase the variability between the simulations and thus more completely include the "true" porosity log data. However, while doing so, this approach does not decrease the mean absolute residual between predicted and measured porosity.

\subsection{Information Obtained From the Porosity Simulations}

[32] To evaluate prediction of the overall 3-D porosity distribution at the BHRS, we ran 100 SA-based simulations using all 31 crosshole GPR data sets together and all the collocated porosity log data. Figure 8 shows a 3-D view of one randomly selected simulation at profiles B6-B1, B1-B2, B2-B3 and B3-C3 with horizontal slices at several depths. Figure 9 shows three randomly chosen realizations at profile A1-B2, and the mean and standard deviation of the 100 simulations. The simulations appear realistic and provide a reliable 3-D estimation of the porosity distribution (Figure 8); comparison of Figure 9 to Figure 2 shows that the relatively large-scale structures in the GPR data have been reproduced in the conditional simulation, as has the small-scale variability through the spatial correlation functions and porosity log data. Furthermore the variability in the simulated porosity values is consistently small at the wells and increases with distance from the wells, and more so where not along a GPR profile. Also, continuity is well constrained between locations that are conditioned differently (i.e., either with well log data, GPR profiles, or without conditioning data).

[33] Finally, the 3-D simulations of porosity using GPR velocity tomograms and porosity $\log$ data (Figures 8 and 9) provide very clear imaging of the: (1) sand channel (Unit 5) thickening toward the river at the top of the simulation, (2) relatively high variability in values and shorter lateral continuity in Units 2 a and 4, (3) relatively high lateral continuity and lower variability in Unit 3, and (4) boundaries between the main units at the BHRS. These imaged details are in agreement with previous geostatistical analysis of the porosity log data at this site [Barrash and Clemo, 2002].

\section{Discussion and Conclusions}

[34] The main objectives of this study were to develop modeling approaches to generate high-resolution 3-D simulations of hydrologic property (porosity) distributions from

Figure 7. Evaluating predictions of measured porosity values (blue lines) that are withheld from the estimation process and located along crosshole GPR profiles: (a) at well B2 and (b) at well A1. This is done by generating 100 3-D realizations (gray lines) of porosity using crosshole GPR and porosity log data in an SA-based approach and using randomly chosen conditional petrophysical relations by considering the uncertainty in the relation between GPR velocity and porosity (Figure 6). The black lines show the means of all the simulations and the red lines show the $95 \%$ intervals of occurrence. Frequency distributions of the correlation coefficients and of the mean absolute residuals between each simulation and the measured data are also shown. 


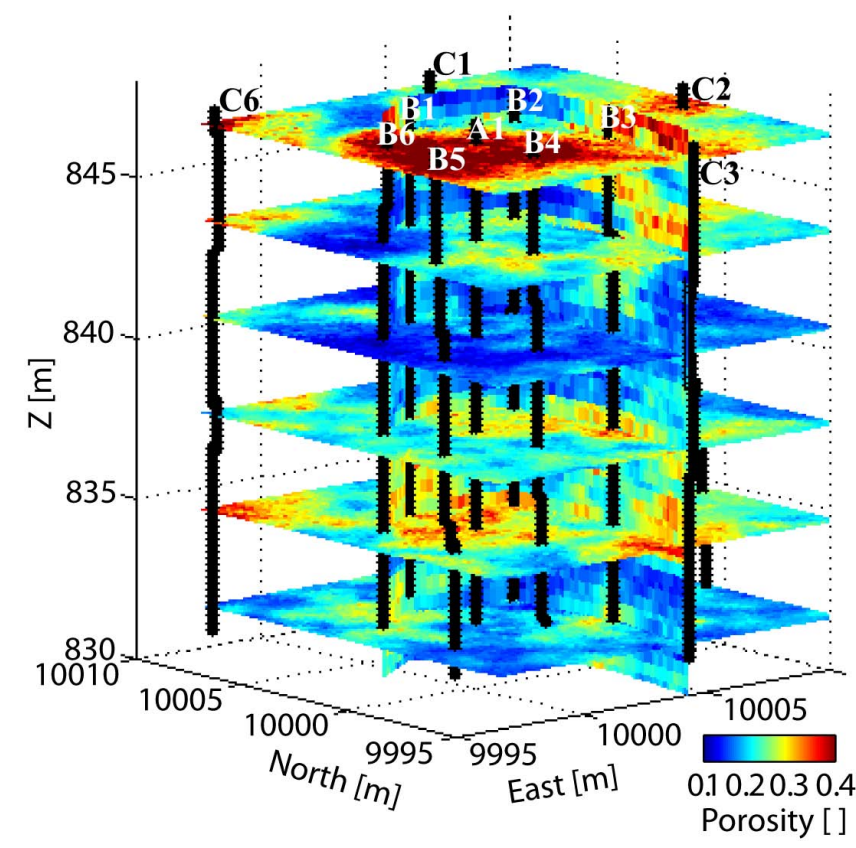

Figure 8. One 3-D realization randomly chosen from 100 , obtained from porosity logs and GPR velocity tomograms using an SA-based approach, and illustrated here at six depths in the aquifer and along profiles B6-B1, B1-B2, B2-B3 and B3-C3.

geophysical (GPR velocity tomography) and hydrologic (porosity log) data, and to evaluate how much benefit results from using GPR tomograms with porosity log data in such simulations. Hence we (1) adapted SA-based and Bayesian sequential simulation approaches to 3-D and for variable spatial data coverage, and (2) investigated the ability of these approaches to predict porosity log data that were withheld from the estimation process (whether located along GPR crosshole profiles or not).

[35] Results show that use of GPR velocity tomograms in the simulation process improved the prediction of porosity compared to using porosity log data only. In particular, by doing this (1) the fit between predicted and "true" porosity is improved, (2) the range of uncertainty estimated by the prediction is reduced, and importantly (3) the correlation between the estimated and "true" porosity is increased. Furthermore these improvements are not only observed when the predicted porosity log is located along a crosshole GPR profile but also when no supplemental data are present at a given prediction location. Finally, variations within reasonable ranges for parameters involved in the simulations (such as spatial correlation function and conditional petrophysical distribution) influence local details of results to some degree but do not change the above findings. For example using a target lateral spatial correlation range of $12 \mathrm{~m}$ instead of $24 \mathrm{~m}$ (as was used here) leads to (1) small decreases in all the correlation coefficients between the withheld and the simulated porosity logs, (2) slightly more variability between the simulations, and (3) an increase in the benefit of using GPR data.

[36] Ultimately, the degree to which GPR data can improve the prediction of porosity is dependent on how closely the geophysically imaged variations are related to the porosity variations. This can be seen for example with the greater improvement in porosity prediction at B2 than A1, where the correlation coefficient between GPR velocity and porosity is greater at B2 than A1. This is not due to more tomograms intersecting at well B2 than A1 but to the less successful GPR imaging of porosity structures at A1. Thus we note that the improvement in porosity estimation brought by including crosshole GPR data can be reduced
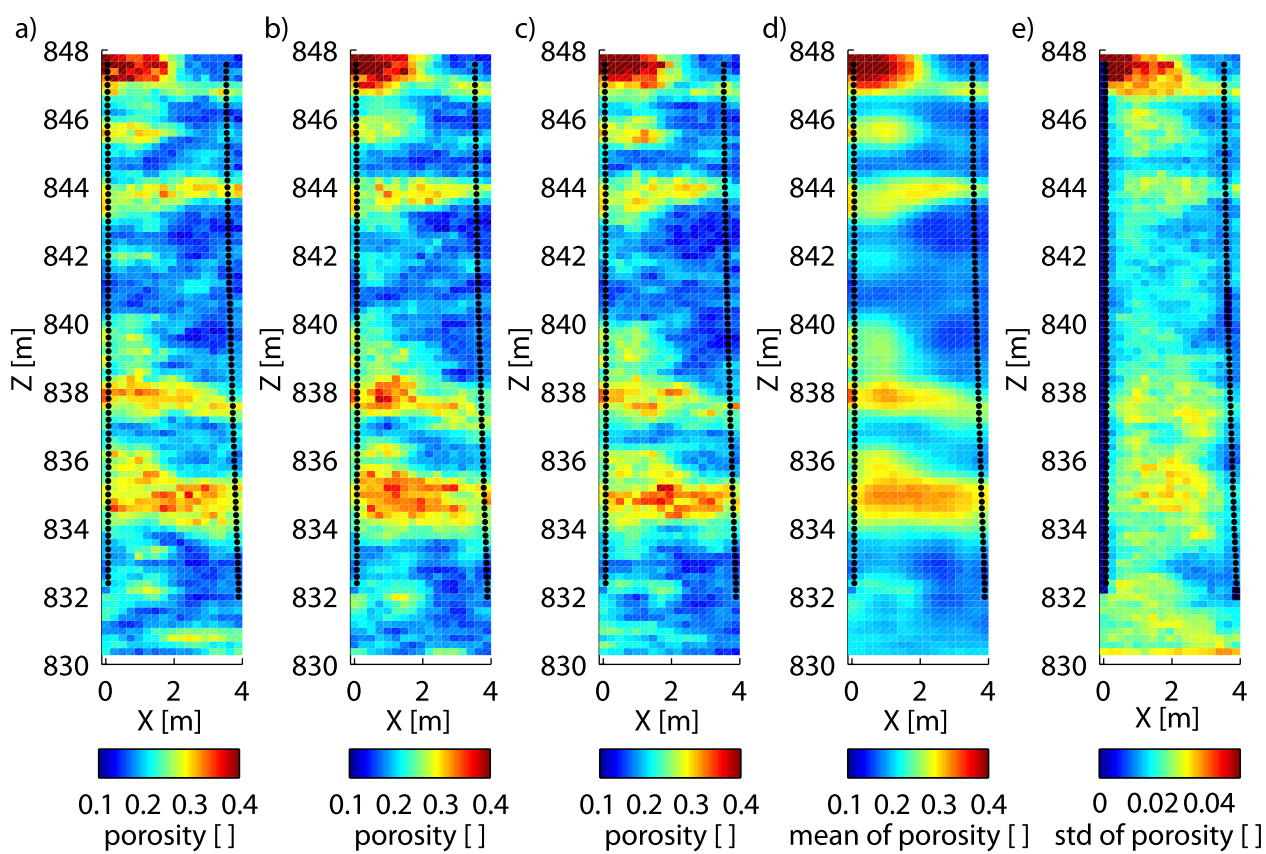

Figure 9. Porosity spatial distributions along profile A1-B2, extracted from 3-D realizations obtained from porosity logs and GPR velocity tomograms using an SA-based approach: (a-c) three randomly chosen realizations, (d) mean and (e) standard deviation of 100 porosity simulations. 
due to (1) limited sensitivity to variations in and between some hydrologic units because of petrophysical behavior, (2) limited resolution depending on the acquisition geometry and physics of the GPR method, and/or (3) artifacts in the GPR tomogram. This means that the geophysical data may be devoid of value, or even possibly counter-productive, in some cases. Possible ways to improve the results in such cases include (1) consideration of the spatially variable nature of the relation between porosity and geophysical data, (2) use of supplementary sources of information such as petrophysics, amplitude tomography, or electrical tomography to perhaps improve the characterization of units where GPR velocity tomography failed, and (3) future developments in the inversion of multiple GPR profiles such as with full waveform inversion.

[37] The SA-based and Bayesian sequential simulations provided quite similar results and so either may perform well depending on the objectives and requirements of a given study. The main difference between the methods as used here is that Bayesian sequential simulation gives more weight to the porosity log data, which can either improve or weaken the result compared to the SA-based approach. Also Bayesian sequential simulation is faster while the SAbased approach allows more flexibility in accounting for different constraints through the use of an objective function. Importantly both show significant flexibility in handling the crucial step of linking the geophysical data to the hydrologic property. And, we did not observe significant changes in results due to the difference in how the spatial correlation function is applied in the two approaches. Indeed, both approaches generate realizations where the experimental spatial functions reproduce well the parametric ones used in the simulation process.

[38] Finally, this study provides reliable high-resolution simulation of the 3-D distribution of porosity in a heterogeneous aquifer at a real field site, the BHRS. As such, these results can be used further in examining hydrologic characterization and hydrogeophysical relations to support hydrologic applications. A next step will be to implement such porosity reconstruction approaches in more global strategies to predict flow and solute transport, and to evaluate the benefits of doing so. This should also allow improvement of our understanding of the relation between various geophysical and/or hydrologic properties in heterogeneous aquifers at the field scale.

[39] Acknowledgments. This research was supported by funding to B Dafflon from the Swiss National Science Foundation and Boise State University. W. Barrash was supported by EPA under grants X-96004601-0 and X-96004601-1, and by the U.S. RDECOM ARL Army Research Office under grant W911NF-09-1-0534. The authors would like to thank the various researchers responsible for collecting and archiving the BHRS data sets used in this analysis. This paper was significantly improved as a result of the constructive comments and suggestions from the reviewers, Associate Editor, and Editor.

\section{References}

Asprion, U., and T. Aigner (1999), Towards realistic aquifer models: threedimensional georadar surveys of Quaternary gravel deltas (Singen Basin, SW Germany), Sediment. Geol., 129(3-4), 281-297.

Bachrach, R. (2006), Joint estimation of porosity and saturation using stochastic rock-physics modeling, Geophysics, 71(5), O53-O63.
Barrash, W., and T. Clemo (2002), Hierarchical geostatistics and multifacies systems: Boise Hydrogeophysical Research Site, Boise, Idaho, Water Resour. Res., 38(10), 1196, doi:10.1029/2002WR001436.

Barrash, W., and E. C. Reboulet (2004), Significance of porosity for stratigraphy and textural composition in subsurface, coarse fluvial deposits: Boise Hydrogeophysical Research Site, Geol. Soc. Am. Bull., 116(9-10), 1059-1073.

Barrash, W., T. Clemo, and M. D. Knoll (1999), Boise Hydrogeophysical Research Site (BHRS): Objectives, design, initial geostatistical results, Proceedings of SAGEEP99, Symposium on the Application of Geophysics to Engineering and Environmental Problems, pp. 389-398, Environmental and Engineering Geophysical Society, Oakland, Calif.

Beres, M., A. Green, P. Huggenberger, and H. Horstmeyer (1995), Mapping the architecture of glaciofluvial sediments with 3-dimensional georadar, Geology, 23(12), 1087-1090.

Cardiff, M., W. Barrash, M. Thoma, and B. Malama (2011), Information content of slug tests for estimating hydraulic properties in realistic, highconductivity aquifer scenarios, J. Hydrol., 403(1-2), 66-82.

Chen, J. S., S. Hubbard, and Y. Rubin (2001), Estimating the hydraulic conductivity at the South Oyster Site from geophysical tomographic data using Bayesian techniques based on the normal linear regression model, Water Resour. Res., 37(6), 1603-1613.

Corbeanu, R. M., G. A. McMechan, R. B. Szerbiak, and K. Soegaard (2002), Prediction of 3-D fluid permeability and mudstone distributions from ground-penetrating radar (GPR) attributes: Example from the Cretaceous Ferron Sandstone Member, east-central Utah, Geophysics, 67(5), 1495-1504.

Dafflon, B., J. Irving, and K. Holliger (2009a), Simulated-annealing-based conditional simulation for the local-scale characterization of heterogeneous aquifers, J. Appl. Geophys., 68(1), 60-70.

Dafflon, B., J. Irving, and K. Holliger (2009b), Use of high-resolution geophysical data to characterize heterogeneous aquifers: Influence of data integration method on hydrological predictions, Water Resour. Res., 45, W09407, doi:10.1029/2008WR007646.

Dafflon, B., J. Irving, and K. Holliger (2010), Calibration of high-resolution geophysical data with tracer test measurements to improve hydrological predictions, Adv. Water Resour., 33(1), 55-68.

Dafflon, B., J. Irving, and W. Barrash (2011a), Inversion of multiple intersecting high-resolution crosshole GPR profiles for hydrological characterization at the Boise Hydrogeophysical Research Site, J. Appl. Geophys., 73, 305-314.

Dafflon, B., W. Barrash, M. Cardiff, and T. C. Johnson (2011b), Hydrological parameter estimations from a conservative tracer test with variabledensity effects at the Boise Hydrogeophysical Research Site, Water Resour. Res., 47, W12513, doi:10.1029/2011WR010789.

Day-Lewis, F. D., and J. W. Lane (2004), Assessing the resolution-dependent utility of tomograms for geostatistics, Geophys. Res. Lett., 31, L07503, doi:10.1029/2004GL019617.

Day-Lewis, F. D., P. A. Hsieh, and S. M. Gorelick (2000), Identifying fracture-zone geometry using simulated annealing and hydraulic-connection data, Water Resour. Res., 36(7), 1707-1721.

Day-Lewis, F. D., J. W. Lane, J. M. Harris, and S. M. Gorelick (2003), Time-lapse imaging of saline-tracer transport in fractured rock using difference-attenuation radar tomography, Water Resour. Res., 39(10), 1290, doi:10.1029/2002WR001722.

Deutsch, C. V. (2002), Geostatistical Reservoir Modeling, 384 pp., Oxford Univ. Press, New York.

Deutsch, C. V., and A. G. Journel (1998), GSLIB: Geostatistical Software Library and User's Guide, 384 p., Oxford University Press, New York.

Deutsch, C. V., and X. H. Wen (1998), An improved perturbation mechanism for simulated annealing simulation, Math. Geol., 30(7), 801-816.

Deutsch, C. V., and X. H. Wen (2000), Integrating large-scale soft data by simulated annealing and probability constraints, Math. Geol., 32(1), 49-67.

Dubreuil-Boisclair, C., E. Gloaguen, D. Marcotte, and B. Giroux (2011), Heterogeneous aquifer characterization from ground-penetrating radar tomography and borehole hydrogeophysical data using nonlinear Bayesian simulations, Geophysics, 76(4), 1-13.

Ezzedine, S., Y. Rubin, and J. S. Chen (1999), Bayesian method for hydrogeological site characterization using borehole and geophysical survey data: Theory and application to the Lawrence Livermore National Laboratory Superfund site, Water Resour. Res., 35(9), 2671-2683.

Gastaldi, C., D. Roy, P. Doyen, and L. Den Boer (1998), Using Bayesian simulations to predict reservoir thickness under tuning conditions, Leading Edge, 17(4), 539-539. 
Gelman, A., J. B. Carlin, H. S. Stern, and D. B. Rubin (2003), Bayesian Data Analysis, 668 pp., Chapman \& Hall/CRC, Boca Raton, Fla.

Goovaerts, P. (1997), Geostatistics for Natural Resources Evaluation, 496 pp., Oxford Univ. Press, New York.

Harp, D. R., Z. X. Dai, A. V. Wolfsberg, J. A. Vrugt, B. A. Robinson, and V. V. Vesselinov (2008), Aquifer structure identification using stochastic inversion, Geophys. Res. Lett., 35, L08404, doi:10.1029/2008GL033585.

Hassan, A. E. (2001), Water flow and solute mass flux in heterogeneous porous formations with spatially random porosity, J. Hydrol., 242(1-2), 1-25.

Hearst, J. R., and P. H. Nelson (1985), Well Logging for Physical Properties, McGraw-Hill, New York.

Hu, B. X., M. M. Meerschaert, W. Barrash, D. W. Hyndman, C. M. He, X. Y. Li, and L. J. Guo (2009), Examining the influence of heterogeneous porosity fields on conservative solute transport, J. Contam. Hydrol., 108(3-4), 77-88.

Hubbard, S. S., and Y. Rubin (2005), Introduction to hydrogeophysics, in Hydrogeophysics, edited by Y. Rubin and S. S. Hubbard, pp. 3-21, Springer, Dordrecht, Netherlands.

Hubbard, S. S., J. S. Chen, J. Peterson, E. L. Majer, K. H. Williams, D. J. Swift, B. Mailloux, and Y. Rubin (2001), Hydrogeological characterization of the South Oyster Bacterial Transport Site using geophysical data, Water Resour. Res., 37(10), 2431-2456.

Hyndman, D. W., J. M. Harris, and S. M. Gorelick (2000), Inferring the relation between seismic slowness and hydraulic conductivity in heterogeneous aquifers, Water Resour. Res., 36(8), 2121-2132.

Irving, J. D., M. D. Knoll, and R. J. Knight (2007), Improving crosshole radar velocity tomograms: A new approach to incorporating high-angle traveltime data, Geophysics, 72(4), J31-J41.

Johnson, T. C., P. S. Routh, W. Barrash, and M. D. Knoll (2007), A field comparison of Fresnel zone and ray-based GPR attenuation-difference tomography for time-lapse imaging of electrically anomalous tracer or contaminant plumes, Geophysics, 72(2), G21-G29.

Kelkar, M., and G. Perez (2002), Applied Geostatistics for Reservoir Characterization, 264 pp., Soc. of Pet. Eng., Richardson, Tex.

Kemna, A., J. Vanderborght, B. Kulessa, and H. Vereecken (2002), Imaging and characterisation of subsurface solute transport using electrical resistivity tomography (ERT) and equivalent transport models, J. Hydrol., 267(3-4), 125-146.

Kowalsky, M. B., S. Finsterle, J. Peterson, S. Hubbard, Y. Rubin, E. Majer, A. Ward, and G. Gee (2005), Estimation of field-scale soil hydraulic and dielectric parameters through joint inversion of GPR and hydrological data, Water Resour. Res., 41, W11425, doi:10.1029/2005WR004237.
Li, L., H. Zhou, J. J. Gómez-Hernández, and H.-J. Hendricks Franssen (2012), Jointly mapping hydraulic conductivity and porosity by assimilating concentration data via ensemble Kalman filter, J. Hydrol., 428 $429,152-169$.

Linde, N., S. Finsterle, and S. Hubbard (2006), Inversion of tracer test data using tomographic constraints, Water Resour. Res., 42, W04410, doi:10.1029/2004WR003806.

Linde, N., A. Revil, A. Boleve, C. Dages, J. Castermant, B. Suski, and M. Voltz (2007), Estimation of the water table throughout a catchment using self-potential and piezometric data in a Bayesian framework, J. Hydrol., 334(1-2), 88-98.

McKenna, S. A., and E. P. Poeter (1995), Field example of data fusion in site characterization, Water Resour. Res., 31(12), 3229-3240.

Moysey, S., K. Singha, and R. Knight (2005), A framework for inferring field-scale rock physics relationships through numerical simulation, Geophys. Res. Lett., 32, L08304, doi:10.1029/2004GL022152.

Mwenifumbo, C. J., W. Barrash, and M. D. Knoll (2009), Capacitive conductivity logging and electrical stratigraphy in a high-resistivity aquifer, Boise Hydrogeophysical Research Site, Geophysics, 74(3), E125-E133.

Paasche, H., J. Tronicke, K. Holliger, A. G. Green, and H. Maurer (2006), Integration of diverse physical-property models: Subsurface zonation and petrophysical parameter estimation based on fuzzy c-means cluster analyses, Geophysics, 71(3), H33-H44.

Scales, J. A. (1987), Tomographic Inversion via the Conjugate-Gradient Method, Geophysics, 52(2), 179-185.

Scheibe, T. D., and Y. J. Chien (2003), An evaluation of conditioning data for solute transport prediction, Ground Water, 41(2), 128-141.

Schön, J. H. (2004), Physical Properties of Rocks : Fundamentals and Principles of Petrophysics, 583 pp., Elsevier, Oxford, UK.

Singha, K., and S. M. Gorelick (2005), Saline tracer visualized with threedimensional electrical resistivity tomography: Field-scale spatial moment analysis, Water Resour. Res., 41, W05023, doi:10.1029/2004WR003460.

Straface, S., F. Chidichimo, E. Rizzo, M. Riva, W. Barrash, A. Revil, M. Cardiff, and A. Guadagnini (2011), Joint inversion of steady-state hydrologic and self-potential data for 3D hydraulic conductivity distribution at the Boise Hydrogeophysical Research Site, J. Hydrol., 407(1-4), 115-128.

Tronicke, J., and K. Holliger (2005), Quantitative integration of hydrogeophysical data: Conditional geostatistical simulation for characterizing heterogeneous alluvial aquifers, Geophysics, 70(3), H1-H10.

Wand, M. P., and M. C. Jones (1995), Kernel Smoothing, Chapman and Hall, New York. 